2008, 112, 9565-9567

Published on Web 07/22/2008

\title{
Immersion of Charged Nanoparticles in a Salt Solution/Air Interface
}

\author{
Prajnaparamita Dhar, ${ }^{\dagger}$ Vikram Prasad, Eric R. Weeks, $₫$ Thomas Bohlein, ${ }^{\S}$ and \\ Thomas M. Fischer*,\$ \\ Department of Chemical Engineering, The University of California Santa Barbara, Department of Physics, \\ Emory University, Atlanta, GA, and Institut für Experimentalphysik V, Universität Bayreuth, 95440 Bayreuth, \\ Germany
}

Received: June 09, 2008; Revised Manuscript Received: July 03, 2008

\begin{abstract}
Electrostatic interactions strongly affect the immersion depth of nanoparticles into an interface. We prove this statement by measuring the diffusion constant of charged nanoparticles at a sodium chloride solution/air interface. Interfacial diffusion of nanoparticles slows down with increasing ionic strength of the sodium chloride solution. Hydrodynamic calculations are used to estimate the immersion depth from the diffusion constant, suggesting that nanoparticles with a carboxylate surface are only slightly immersed into a bare air/water interface. With increasing molarities of sodium chloride, the immersion depth increases to complete immersion for a $10^{-2}$ molar solution. Our experiments show that the location of nanoparticles at interfaces is determined by an intricate interplay between the electrostatic properties of the solution/air interface, the solution/solid interface, and the classical contact angle.
\end{abstract}

\section{Introduction}

The mobility of colloidal particles as tracer particles is useful for studying the rheological properties of complex materials; this is termed "microrheology". ${ }^{1}$ Microrheology is a powerful tool for the indirect detection of length scales such as hydrodynamic radii, length scales of confinement, or heterogeneities in three-dimensional complex fluids. The deduction of those length scales from the observed nanoparticle trajectories, however, depends on microrheological models of the viscoelastic surroundings traced by the particles. The more complex the structure of the fluid, the more complex are the assumptions of the model, resulting in more difficulties in checking their validity. Prominent examples of an apparently simple fluid environment are an air/liquid interface or liquid/liquid interface. Despite the apparent simplicity, there is dispute about the arrangement of charged nanoparticles into an air/water interface ${ }^{2-6}$ that has been triggered by the observation of anomalous longrange attractions between interfacial charged nanoparticles. ${ }^{7-9}$ It has been suggested that the energy of the electrostatic field generated by the particles could be reduced by a deeper immersion of the nanoparticles into the water, where ions screen the electrostatic field. ${ }^{9}$ The immersion would be at the cost of surface energy produced by the larger area of the now deformed air/water interface. Theoretical arguments based on modeling the water as a structureless dielectric liquid suggest that such electrodipping effects are too weak to cause substantial change of immersion of the particle. ${ }^{3,6}$ It is, however, long known that water is not a structureless fluid and that the structure of water changes near the interface with a slight preference of the

* To whom correspondence should be addressed. E-mail: thomas.fischer@uni-bayreuth.de.

$\dagger$ The University of California.

* Emory University.

$\S$ Universität Bayreuth. hydrogen atoms of the water molecules to point toward the exterior, ${ }^{10,11}$ giving rise to an electrostatic surface potential of the interface. The Angstrom range of the interfacial structure of water protects the surface potential from being screened by the addition of salt. Sometimes the surface potential may even be enhanced by the addition of salt. It is therefore not straightforward to discard electrodipping effects in a theoretical treatment of the immersion of nanoparticles.

In this Letter, we report on the effects of electrostatic interactions on the depth of immersion of charged beads at an air/salt solution interface. Electrostatic interactions have been shown to play a dominant role in the case of charged colloidal particle suspensions at oil-water interfaces. ${ }^{9,12}$ The diffusion of single nanoparticles is used to experimentally measure the translational drag and fit by a modified Stokes-Einstein formula from which the immersion depth is calculated. The electrostatic forces on the spheres are altered by changing the electrolyte concentration of the bulk fluid. The results suggest that the immersion depth changes with the electrolyte concentration of the bulk fluid reservoir to which the interface is coupled, suggesting that the electrostatic forces on the sphere contribute to the immersion.

We use standard particle tracking methods ${ }^{13}$ to follow the motion of the tracer particles and obtain their mean square displacement at the salt solution/air interface. We used fluorescent carboxylate modified $\left(0.35 \mathrm{COOH}\right.$ groups $\left./ \mathrm{nm}^{2}\right)$ nanoparticles of radius $a=85 \mathrm{~nm}$ dispersed in an aqueous solution. The solution was passed through an ion-exchange resin and then redispersed in a 20:80 water/isopropanol mixture. Small aliquots of this mixture were spread on ultrapure water of resistivity greater than $18 \mathrm{M} \Omega \cdot \mathrm{cm}$ in a Teflon trough. Data were taken after letting the isopropanol evaporate for $45 \mathrm{~min}$ and after adding different amounts of $\mathrm{NaCl}$, obtained from Fisher Scientific and claimed to be $>99 \%$ pure. Typical conditions 


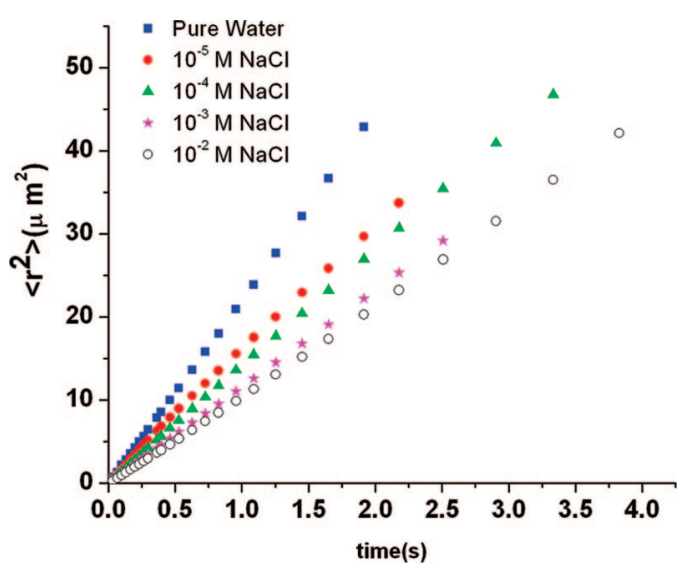

Figure 1. Mean square displacement of carboxylate modified polystyrene nanoparticles on a sodium chloride/air interface as a function of time for different ionic strengths of the solution.

had approximately $N=100$ nanoparticles statistically dispersed on an area of the order $10^{4} \mu \mathrm{m}^{2}$. Images were recorded using a fluorescence microscope (LEICA DM 4000) at $30 \mathrm{fps}$ for a time of approximately $T=50 \mathrm{~s}$.

Within each image, particles are clearly distinguished as bright spots. Particle tracking was done by first locating all particles in each image and then identifying each particle in subsequent images based on proximity to its position in previous images: ${ }^{13}$ in all cases, particles move only small distances compared to their interparticle spacing, so tracking the particles is straightforward. The motion of the particles consisted of random diffusive motion and collective drift induced by the convection of air above the surface. The convective drift velocity was never more than $30 \mu \mathrm{m} / \mathrm{s}$ and was always uniform over the field of view. This velocity was determined by the ensemble average over the velocities of the individual particles, $\mathbf{v}(t)=1 / N \sum_{j}\left[\mathbf{r}_{j}(t\right.$ $\left.+\Delta t)-\mathbf{r}_{j}(t)\right] / \Delta t$. The diffusive particle motion is then computed by measuring the displacement of each particle with the mean drift of all particles being subtracted: $\Delta \mathbf{r}_{i}(t, \tau)=\mathbf{r}_{i}(t+\tau)-$ $\mathbf{r}_{i}(t)-\int_{t}^{t+\tau} \mathbf{v}\left(t^{\prime}\right) \mathrm{d} t^{\prime}$. The diffusive mean square displacement was computed from the time and ensemble average as $\left\langle\Delta r^{2}\right\rangle=$ $1 / N(T-\tau) \sum_{j} \int_{0}^{T-\tau} \Delta r_{j}^{2}(t, \tau) \mathrm{d} t$. The salt solution has a viscosity $\eta$ approximately 50 times more viscous than the air, so the partitioning of the nanoparticle between the fluid and the gaseous phase results in substantial differences in the mobility of the particles.

Figure 1 shows the mean square displacement of the nanoparticles for different ionic strengths of the sodium chloride solution. For each solution, the particles diffuse according to $\left\langle\Delta r^{2}\right\rangle=4 D \tau$ with a diffusion constant $D$ that decreases with increasing ionic strength. The diffusion constant is related to the translational friction coefficient $f$ of the nanoparticle via $f$ $=k_{\mathrm{B}} T / D \eta a$, where $k_{\mathrm{B}}$ is Boltzmann's constant, $T=295 \mathrm{~K}$ is the temperature, and $\eta=0.955 \mathrm{mPa} \cdot \mathrm{s}$ at $22{ }^{\circ} \mathrm{C}$ is the viscosity of water, which remains unaltered by the addition of salt. We find a roughly logarithmic increase of $f$ with the ionic strength, as shown in Figure 2.

The friction coefficient $f$ can be calculated from the knowledge of the rheological properties of the salt solution/air interface, the viscous properties of the solution, and the geometric arrangement of the nanoparticles in the interface. Here, we use the results of Fischer, Heinig, and Dhar, ${ }^{14}$ who calculated the viscous drag coefficient of a partially immersed sphere (immersion depth $d$ ) in an interface. Here, $d$ is the distance of the north pole from the air water interface $(d=0$ corresponding to a sphere touching the air/water interface from

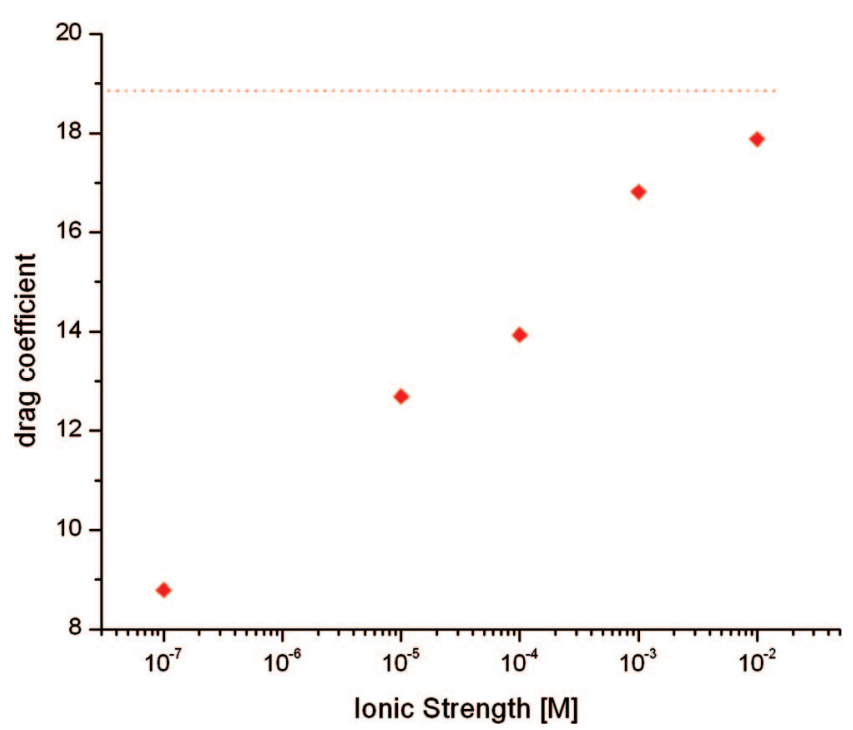

Figure 2. Translational drag coefficient $f$ as a function of the ionic strength of the solution. The dashed line indicates $f(=6 \pi)$ assuming a particle in water with a viscosity of $\eta=0.955 \mathrm{mPa} \cdot \mathrm{s}$ at $22{ }^{\circ} \mathrm{C}$ from the Stokes-Einstein relation for the diffusion of particles in a fluid. The error in the measurement of the drag coefficient is 5\% and arises due to uncertainties in the particle tracking method.

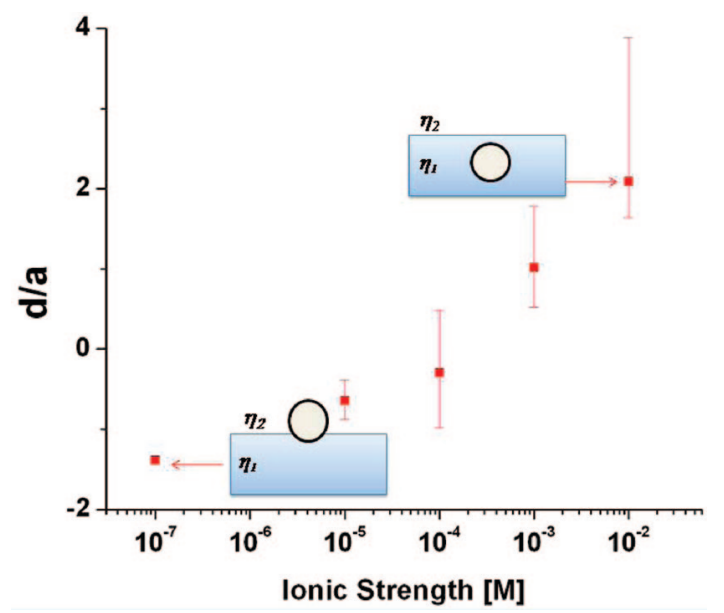

Figure 3. Immersion depth of the nanoparticles as a function of the ionic strength of the solution. Data for $d / a>0$ correspond to particles not touching the interface but rather fully immersed into the liquid, as shown in the top sketch.

below, $d=-a$ an equatorial immersion, $d=-2 a$ corresponding to a sphere that is sitting on top of the interface, and $d>0$ corresponding to a sphere with its north pole lying below the undeformed air/water interface). The interface is assumed to be slightly surfactant contaminated (as indicated by pressure area isotherms for the particles at the interface) and therefore incompressible $\left(\kappa_{\mathrm{s}} \eta a \dot{\gamma} \ll 1\right.$, using $\kappa_{\mathrm{s}}$ the interfacial compressibility and $\dot{\gamma}$ the shear rate), and the interface has a surface viscosity $\eta_{\mathrm{s}}$ and surface tension $\sigma \gg \eta a \dot{\gamma}$. Under these assumptions, the friction coefficient depends on two dimensionless groups. The first group is the ratio of the immersion depth $d$ to the radius $a$ of the nanoparticle $(x=d / a)$, and the second is the Boussinesq number $B=\eta_{\mathrm{s}} / \eta a$. In the current experiments, the Boussinesq number is small, $B \approx 0$, and the theory of Fischer, Heinig, and Dhar predicts an immersion depth $d \approx$ $\left[\left(9 \pi^{2} / 32\right) \operatorname{artanh}\left(f^{2} / 36 \pi^{2}\right)-2\right] a$. In Figure 3 , we plot the immersion depth as a function of the ionic strength.

Figure 3 predicts the nanoparticles to be only slightly immersed into the water at low ionic strength. As the ionic 
strength increases, the immersion increases with an equatorial immersion $d / a=-1$ reached around an ionic strength of $I=$ $10^{-5}$, an increasing immersion in the range between $10^{-4}<I$ $<10^{-3}$, and a complete immersion $d / a>0$ at higher ionic strength. Measurements of the contact angle of water with carboxylate modified particles of larger size reveal no variation of contact angle with the ionic strength. ${ }^{2}$

The $\mathrm{COOH}$ group on the sphere dissociates into $\mathrm{COO}^{-}$and $\mathrm{H}^{+}$in water such that the wetted part of the sphere surface carries a net negative charge. Since our results show a strong dependence of the diffusion constant of the beads on the electrolyte concentration, electrostatic forces may be regarded as a strong contributor to the forces on the nanoparticles at the interface. One would expect that the addition of salt would screen the electrostatic charges on the sphere, resulting in a decrease in the electric forces on the sphere. This would cause a smaller immersion of the spheres into the interface. However, the tendency of the particles to be more deeply immersed into the water at higher ionic strength contradicts the simple assumption that the electrostatic pressure on the nanoparticles is most pronounced when screening is minimal. The observation obviously cannot be understood by arguing with the electrostatic screening in the bulk water. The deviation of the interfacial electrostatics from bulk behavior caused by the orientation order of the water molecules in the outermost layer of the liquid water could be one of the causes of such counterintuitive behavior. The electric pressure on the particle depends on the contrast in surface potential between the air/water interface and the particle. While it is evident from our results that the electrostatic charges contribute to the immersion depth of a sphere at a monolayer, the electrodipping theory in its present form cannot explain the dipping direction obtained in Figure 3.

Future theoretical work, in our opinion, should take into account not only the distribution of ions close to the particle interface but also the orientational ordering of the water molecules close to the air/water interface. An improved theory would also require considering the contrast in the surface potentials at the interface and relate it to a change in the electrostatic interactions at the interface. Therefore, further analysis is needed, taking into account these interactions. It might well be that these are the major reasons for the anomalous mobility of charged interfacial nanoparticles, as well as the cause for anomalous long-range attractions observed between interfacial charged colloids. This may be of special relevance in biology where the membranes are surrounded by water, ions $\left(\mathrm{Na}^{+}, \mathrm{K}^{+}\right)$, and other molecules such that electrostatic effects become significant for the mobility of molecular proteins at interfaces.

Acknowledgment. This material is based upon work supported by the National Science Foundation under CHE-0649427. V.P. and E.R.W. were supported by the National Science Foundation under DMR-0603055.

\section{References and Notes}

(1) Mason, T. G.; Weitz, D. A. Optical Measurements of FrequencyDependent Linear Viscoelastic Moduli of Complex Fluids. Phys. Rev. Lett. 1995, 74, 1250-1253.

(2) Danov, D.; Kralchevsky, P. A.; Boneva, M. P. Electrodipping force acting on a solid particle at a fluid interface. Langmuir 2004, 20, 6139.

(3) Megens, M.; Aizenberg, J. Capillary attraction (communication arising): Like-charged particles at liquid interfaces. Nature 2003, 424, 1014.

(4) Foret, L.; Wuerger, A. Electric-field induced capillary interaction of charged particles at a polar interface. Phys. Rev. Lett. 2004, 92, 5830258306.

(5) Dominguez, A.; Oettel, M.; Dietrich, S. Absence of logarithmic attractions between colloids trapped at the interface of droplets. Europhys. Lett. 2007, 77, 68002.

(6) Oettel, M.; Dietrich, S. Colloidal interactions at fluid interfaces. Langmuir 2008, 24, 1425-1441.

(7) Ruiz-Garcia, J.; Gamez-Corrales, R.; Ivlev, B. I. Formation of two dimensional colloidal voids, soap froths and clusters. Phys. Rev. E 1998, $58,660-663$.

(8) Ruiz-Garcia, J.; Ivlev, B. I. Formation of colloidal clusters and chains at the air/water interface. Mol. Phys. 1998, 95, 371-375.

(9) Nikolaides, M. G.; Bausch, A. R.; Hsu, M. F.; Dinsmore, A. D.; Brenner, M. P.; Gay, C.; Weitz, D. A. Attractive potential between repulsive particles on liquid-liquid interfaces. Nature 2002, 420, 299-301.

(10) Feller, S. E.; Pastor, R. W.; Rojnuckarin, A.; Bogusz, S.; Brooks, B. R. Effect of electrostatic force truncation on interfacial and transport properties of water. J. Phys. Chem. 1996, 100, 17011.

(11) Jungwirth, P.; Tobias, D. J. Ions at the air water interface. J. Phys. Chem. B 2002, 106, 6361-6373. Jungwirth, P.; Tobias, D. J. The molecular structure of salt solution: a new view of the interface with implications for heterogeneous atmospheric chemistry. J. Phys. Chem. B 2001, 105, 1046810472 .

(12) Leunissen, M. E.; van Blaaderen, A.; Hollingsworth, A. D.; Sullivan, M. T.; Chaikin, P. Electrostatics at the oil-water interface, stability, and order in emulsions and colloids. Proc. Natl. Acad. Sci. U.S.A. 2007, 104 (8), 2585-2590.

(13) Crocker, J. C.; Grier, D. G. Methods of digital video microscopy for colloidal studies. J. Colloid Interface Sci. 1996, 179, 298-310.

(14) Fischer, M.; Heinig, P.; Dhar, P. The viscous drag of spheres and filaments moving in membranes or monolayers. J. Fluid Mech. 2006, 558, $451-475$.

JP805042J 